\title{
THE APOLLO 14 MISSION AND PRELIMINARY RESULTS
}

\author{
L. R. SCHERER
}

Director, Apollo Lunar Exploration, Apollo Program Office, National Aeronautics and Space Administration, Washington, D.C., U.S.A.

\begin{abstract}
In February 1971, the Apollo 14 mission successfully landed in the Fra Mauro region of the Moon. Astronauts deployed a sophisticated long-lived geophysical station. While on the surface they performed active seismic and magnetic experiments, deployed a solar wind collector and laser reflector, made an extensive geologic traverse, and performed television, movie, and still camera photography and other minor tasks. They selected a variety of samples of lunar material and returned to Earth over $40 \mathrm{~kg}$ for study. In orbit about the Moon, a variety of scientific and operational photography was accomplished.
\end{abstract}

The initial Apollo missions were directed to smooth mare regions of the Moon. Apollo 14 was the first mission to land in a non-mare area. The Fra Mauro formation is a peculiar geologic unit found in a number of areas around the Imbrium basin. It is thought that this formation represents throw-out resulting from impact of a smaller Moon which formed Imbrium. If this premise is true, material from this region may

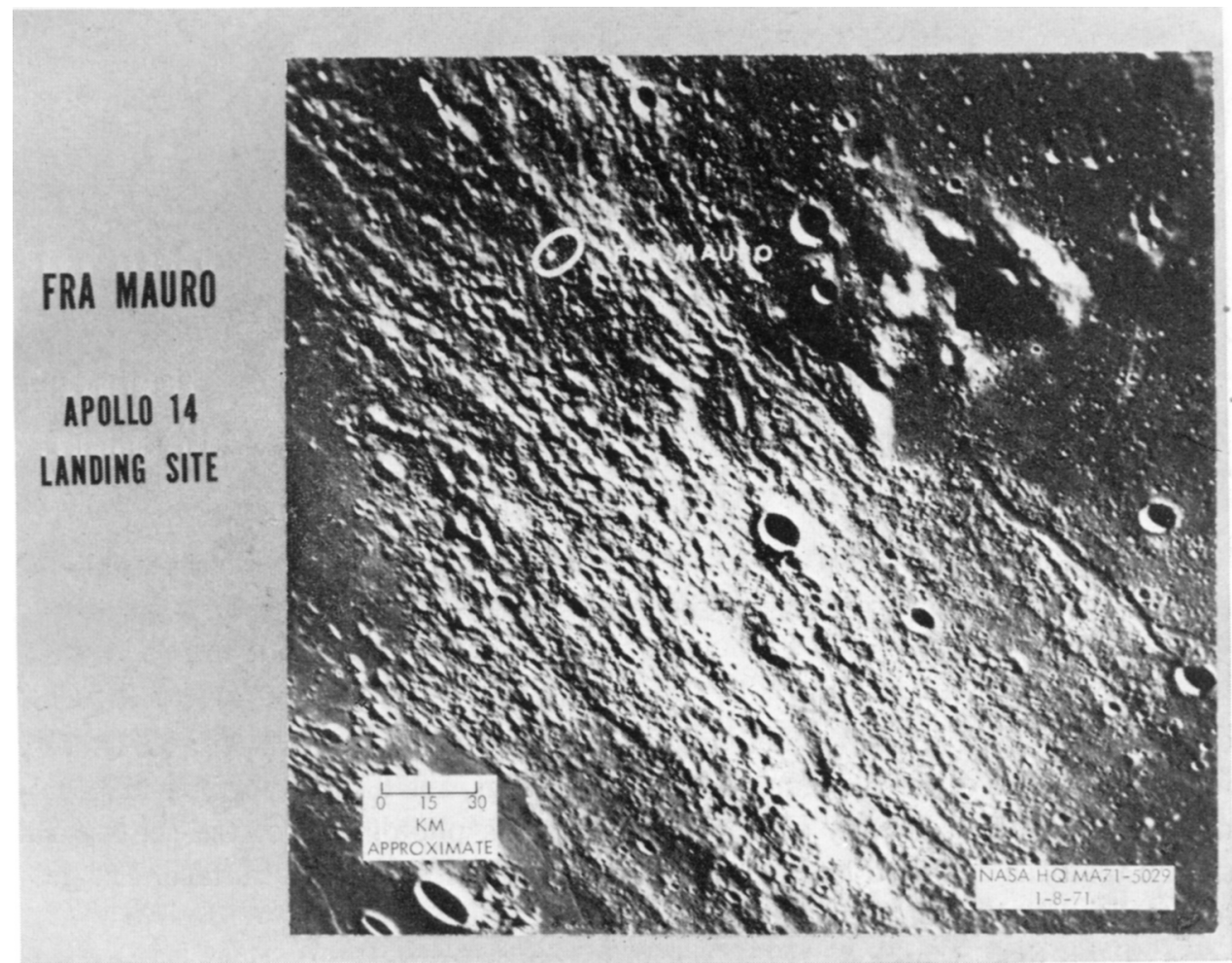

Fig. 1. Medium resolution of Fra Mauro. 
provide a chronology milestone for this major event in the early history of the Moon. The material may represent original crustal material as well as that from deep within the interior. One can see from Figure 1 the apparent radial patterns in the broad area, that seem to come from the Imbrium basin. We selected a specific landing site near a fresh moderate size crater which we concluded would have penetrated the upper surface rubble. It was felt the probability of returning original Fra Mauro material was increased by sampling from the vicinity of the crater rim. The mission proceeded as planned and on February 5, Apollo 14 touched down softly at the prescribed site. For this mission we developed a mobility aid for the crew as shown in Figure 2. As you

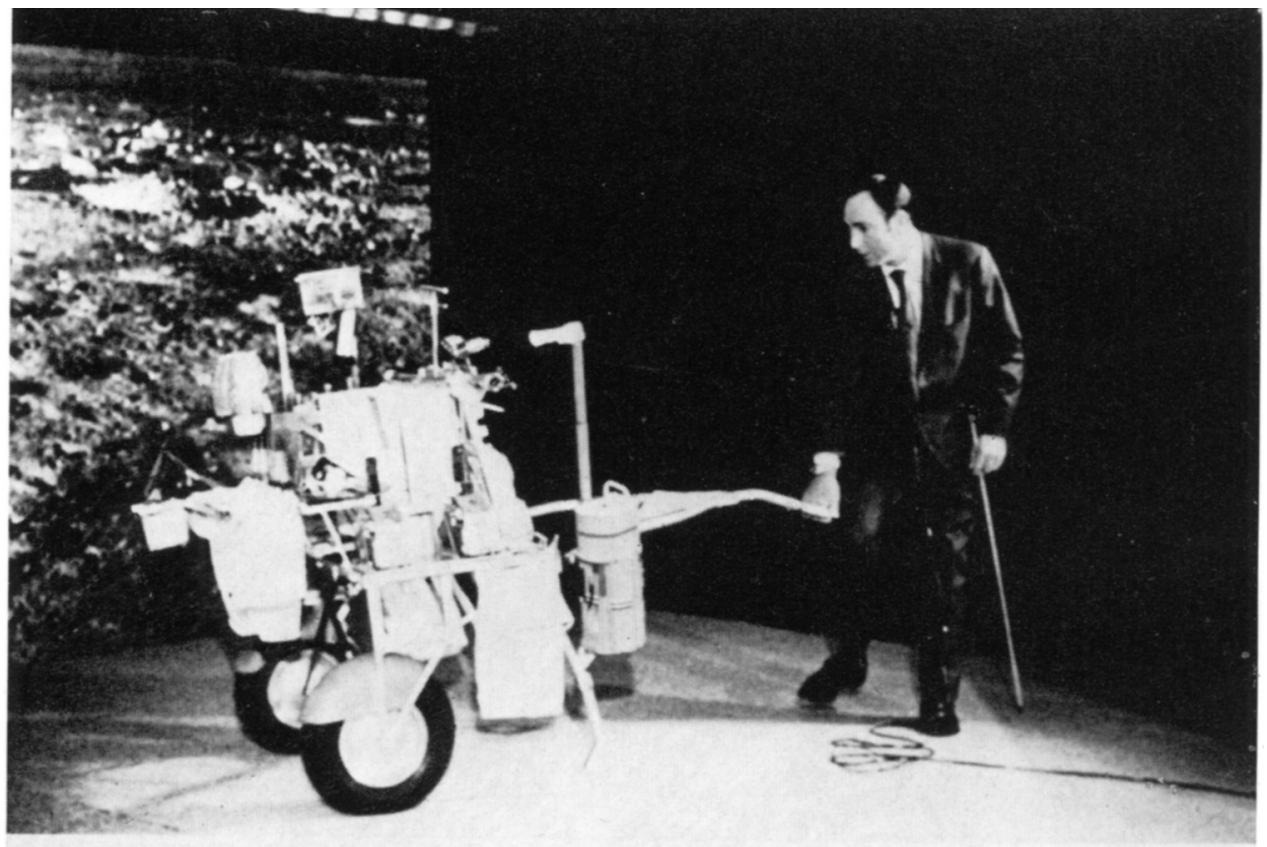

Fig. 2. Mobile Equipment Transporter (MET).

can see from the payload mounted on the little cart, the astronauts were able to carry with them a wide variety of cameras, tools, rock bags, and a small portable magnetometer. It proved to be very effective. Figure 3 is a photograph of the geophysical station that was deployed by the astronauts on the first trip to the surface. The geometry of the deployment is shown on Figure 4.

As of this date, March 23, 1971, scientific results from these various experiments must be considered as very preliminary. The passive seismometer in the first 49 days of 


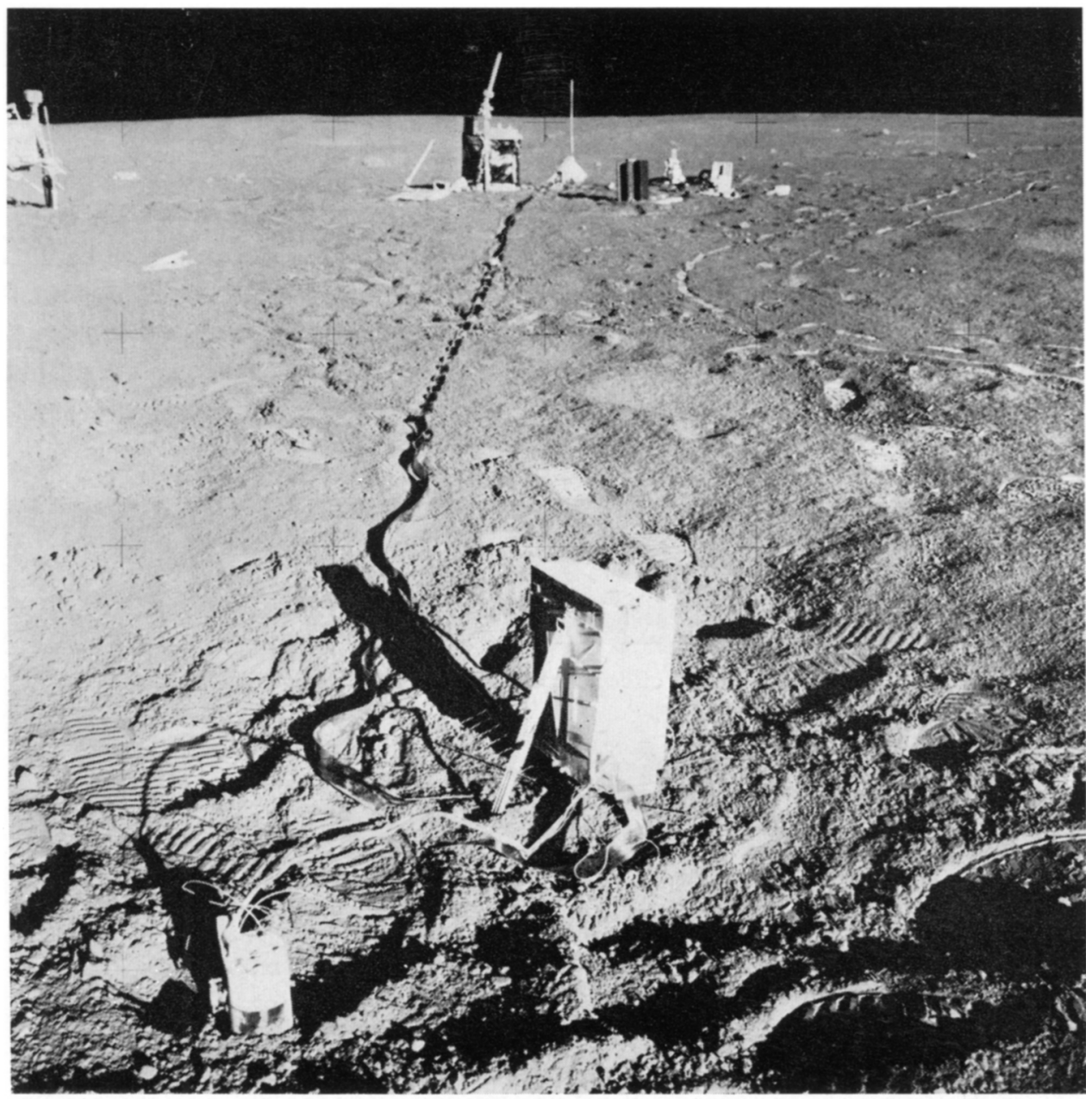

Fig. 3. Geophysical station.

operation has recorded some 60 events. Twenty of these were noted also on the seismometer deployed by Apollo 12 about $181 \mathrm{~km}$ away. We have been recording seismic signals of distinctive characteristics around the time that the Moon and Earth are in closest proximity. These were thought to be the result of tidal strains. The initial thought of the Principal Investigator is that they might be produced in the Fra Mauro crater region but this is a very preliminary guess. During the first perigee after Apollo 14 two of these events were seen on both seismometers. Further analysis may lead to determination of the more precise origin.

For the active seismic experiment, a geophone line $300 \mathrm{ft}$ long was deployed. A total of thirteen energy impacts were placed into the surface by the astronauts with a thumper. The data returned appear to be excellent. The initial estimate is that the 
APOLLO 14 ALSEP LAYOUT

(NOT TO SCALE)
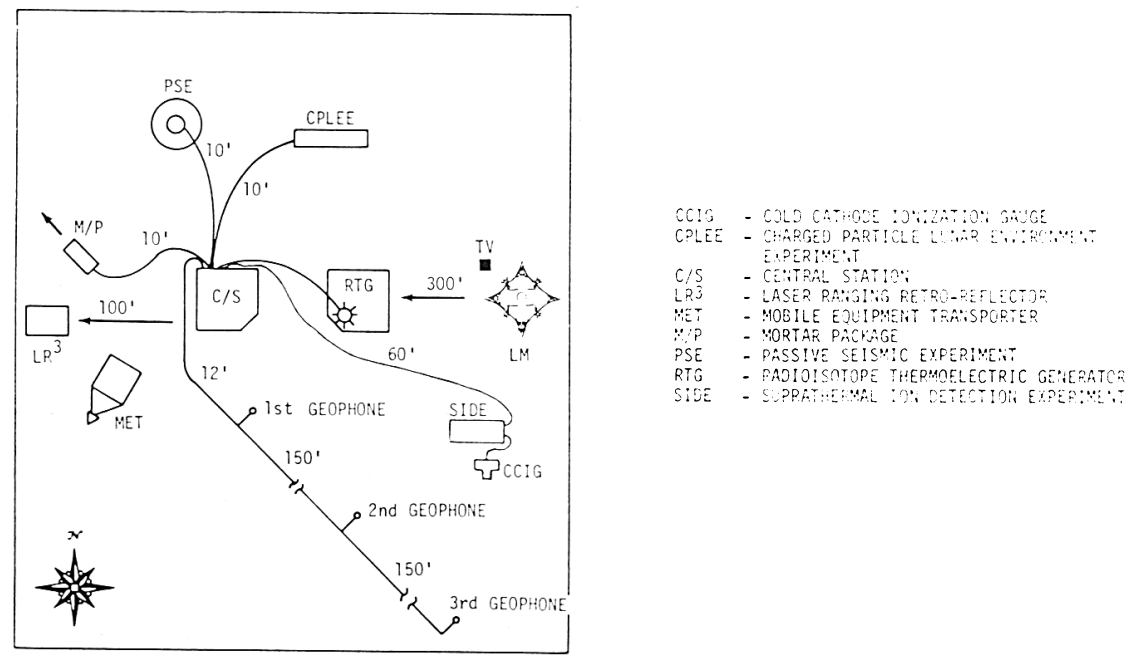

Fig. 4. Geometry of deployment of the geophysical station.

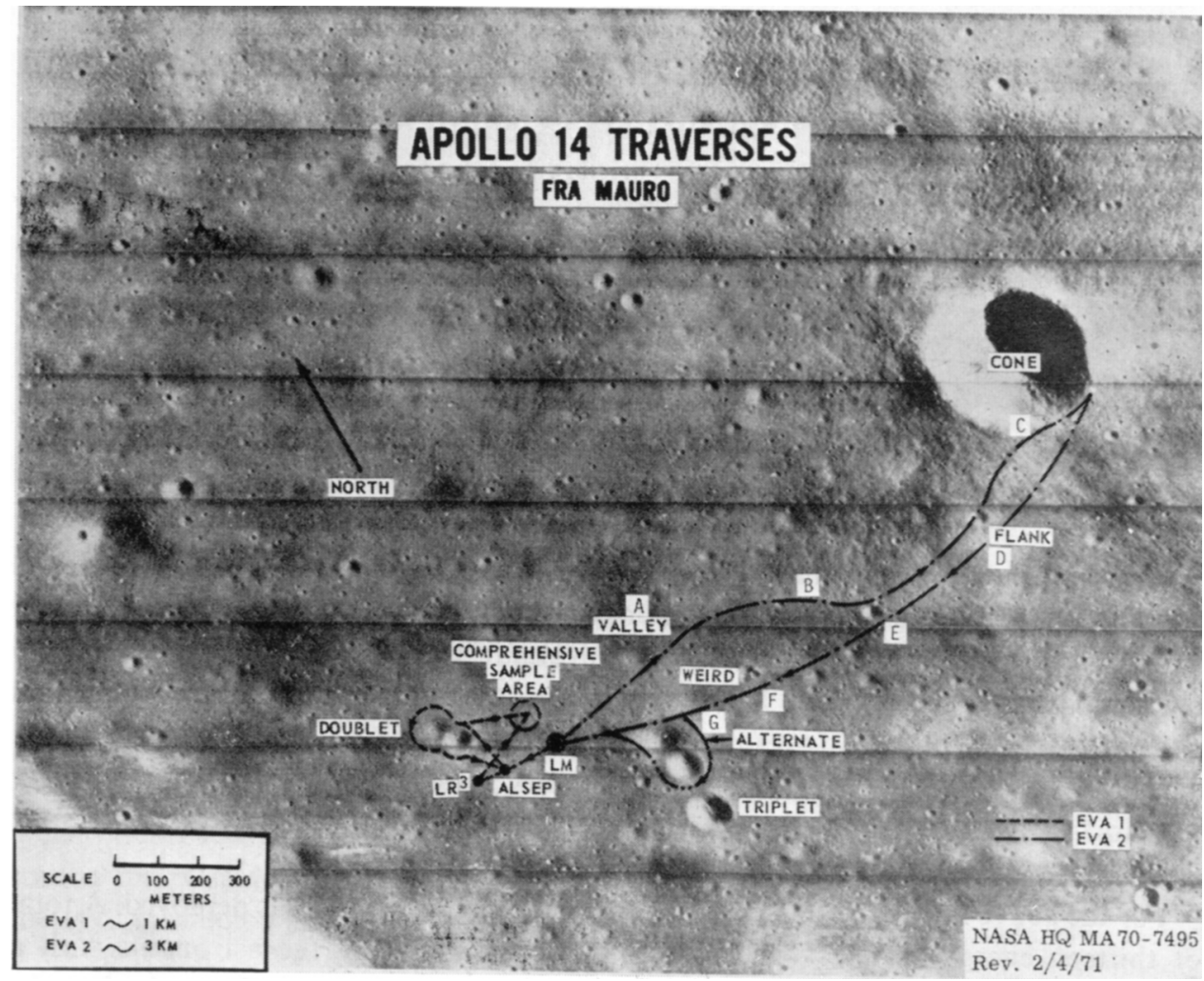

Fig. 5. Apollo 14 traverses. 
surface rubble appears to be about $8 \mathrm{~m}$ deep. There appears to be another layer at 50-70 $\mathrm{m}$ from the data from both seismic instruments.

The Suprathermal Ion Detector has been recording numerous charged particles but no particular analysis has as yet been issued. The Cold Cathode Gauge which measures the intensity of particles has recorded two incidents of sudden increases in pressure which may be indicative of gaseous emissions. On one of these, the pressure increased by a factor of ten for a $1 \mathrm{hr}$ period; on another by a factor of $2-4$ for a $9-\mathrm{hr}$ period. It is too early to interpret the significance of these changes. The Charged Particle Instrument has recorded interesting data as it has passed through the Earth's magnetic tail. It has seen extremely high energy particles greater than $50 \mathrm{keV}$. It has also shown that the electrons seem to be strongly correlated along the tail. The

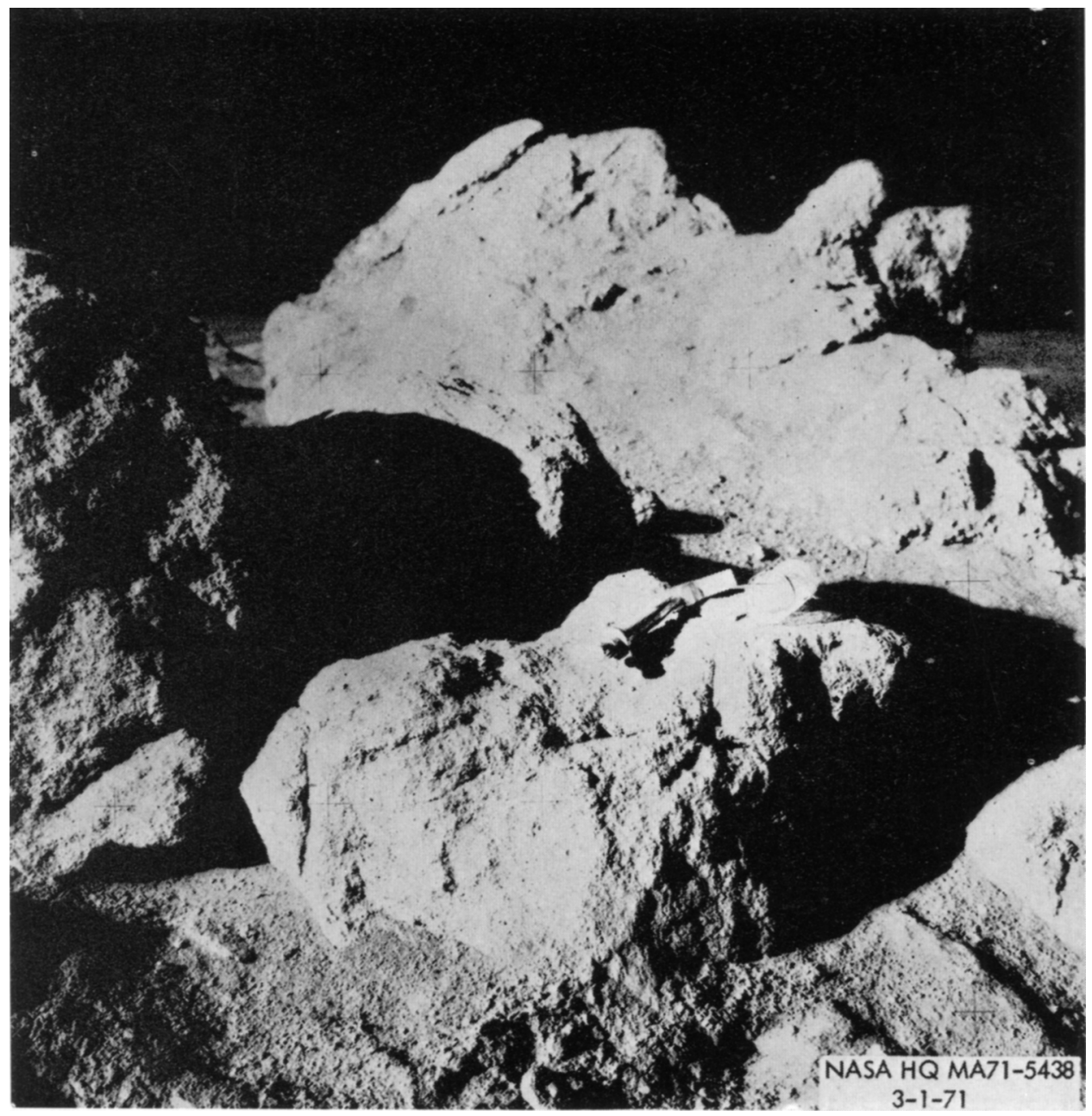

Fig. 6. White rock. 
final instrument deployed by the astronauts was a laser reflector. This was a somewhat improved version over that deployed by the Apollo $11 \mathrm{crew}$. The McDonald Observatory in Texas was able to bounce signals from the reflector while the astronauts were still on the surface.

After a rest period the astronauts emerged for the second surface period. This was devoted to a variety of tasks on a traverse up to the rim of Cone Crater (Figure 5). Not far from the Lunar Module they took their first magnetometer reading which showed about $100 \gamma$ signal. Near the rim of the crater the instrument indicated $40 \gamma$. These readings were considered surprising both in terms of strength and gradient over this short distance. In the vicinity of Cone Crater the astronauts could not determine their precise location due to large boulders and various ridges. When the traverse was

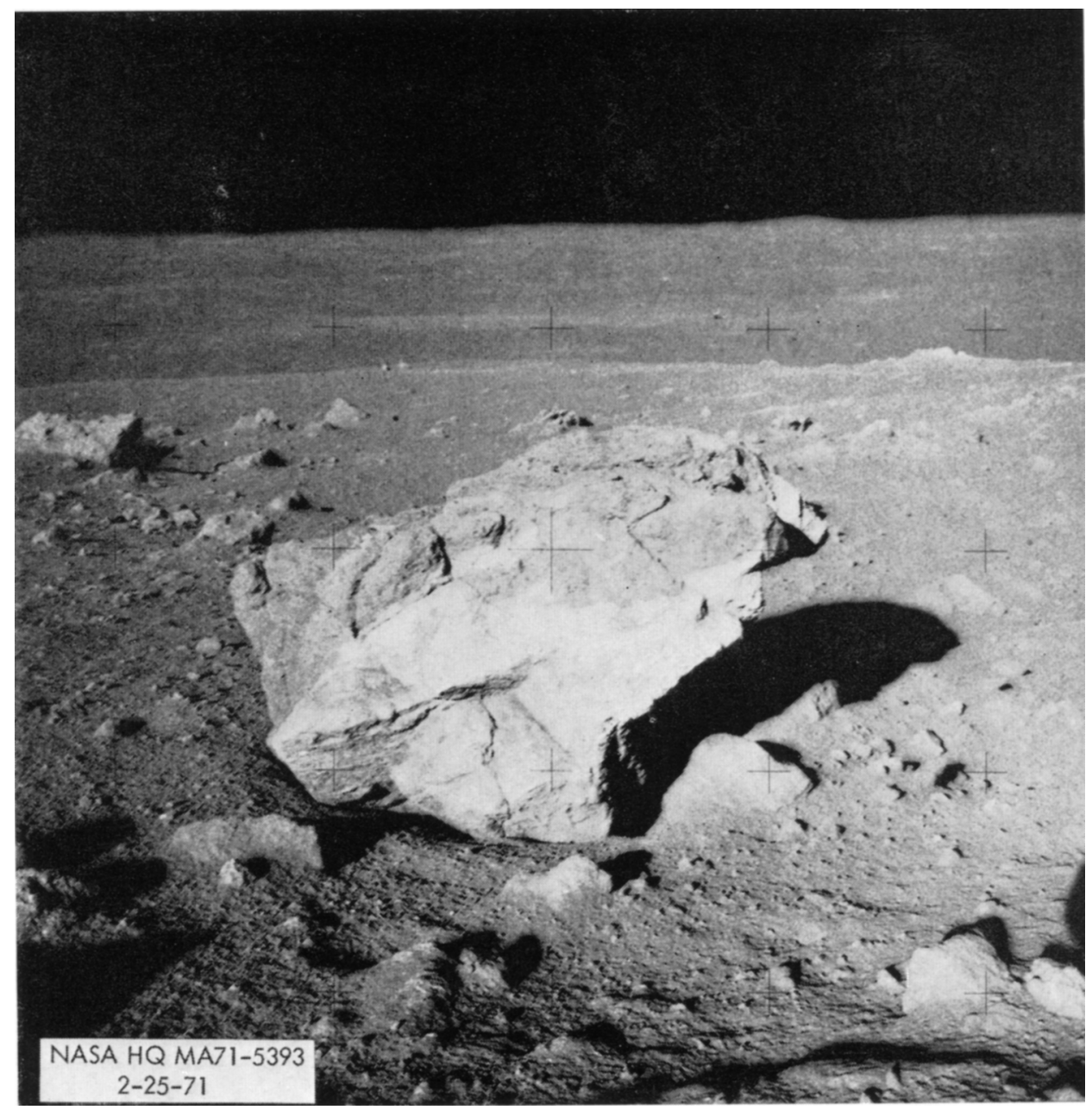

Fig. 7. Multi-colored rock - LM in background. 
reconstructed from returned photography it was shown they were within just a few feet of the rim of the crater. Although it would have been interesting if they could have looked down into the crater to see if any stratification could have been observed, this is not thought to have been a serious scientific omission since the prime objective was to return rocks from the edge of the crater, and this was accomplished. Figure 6 is an interesting white rock on the crater rim from which the astronauts removed and returned a large chip. Nearby, Figure 7, is a peculair multi-colored rock. The landing module can barely be seen in the far distance. Within just a few feet, they found the rock shown in Figure 8 which has a completely different appearance and proved to be much harder than the two previously shown. Figure 9 is a trench that the astronauts dug. As can be seen, the sides collapsed easily. This had not been experienced in previous missions and indicates a much lower cohesiveness of the soil at this site than in the previous mare. The astronauts returned a total of $94 \mathrm{lbs}$ of rocks and soil. Most

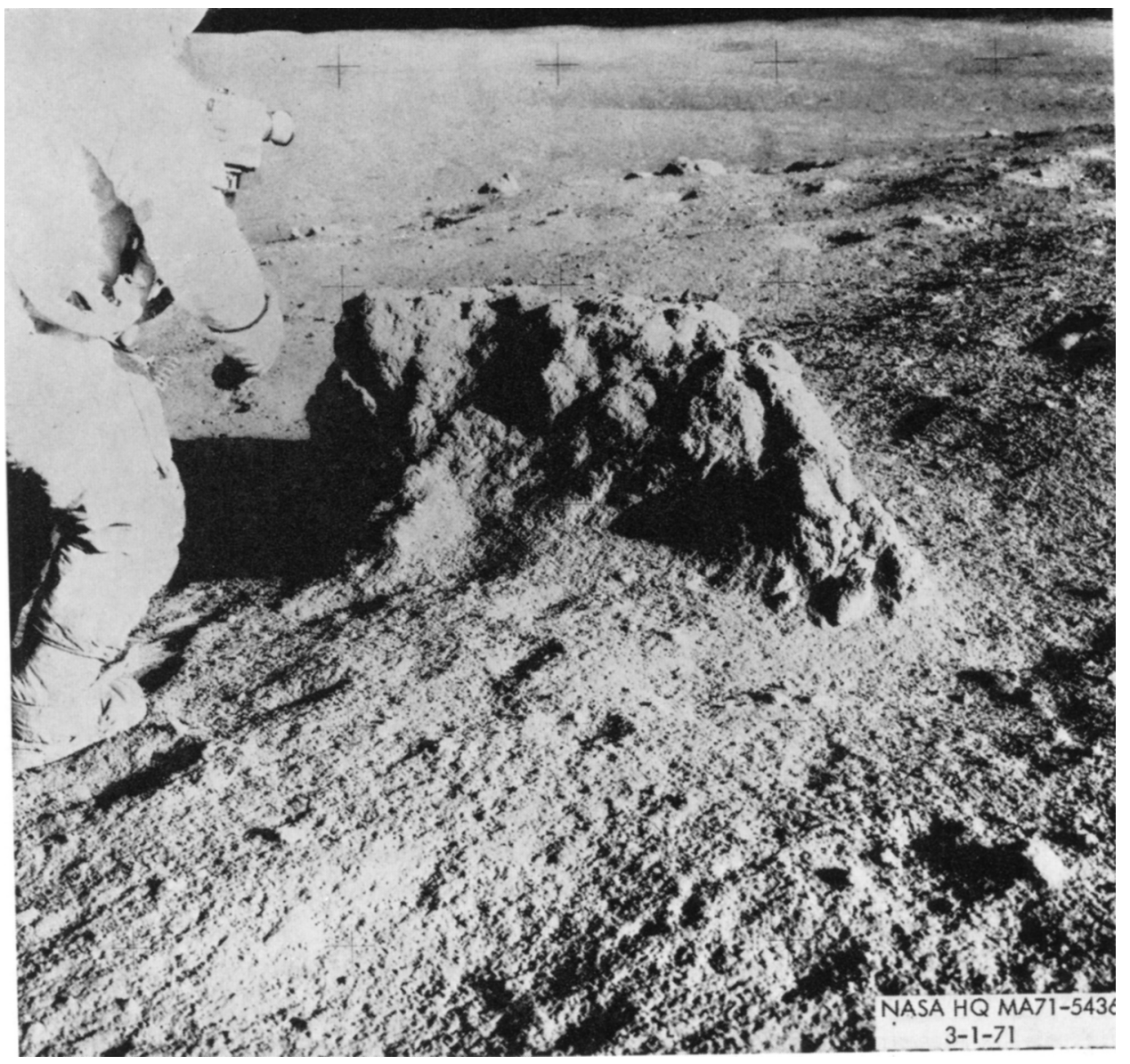

Fig. 8. Hard rock - Igneous. 


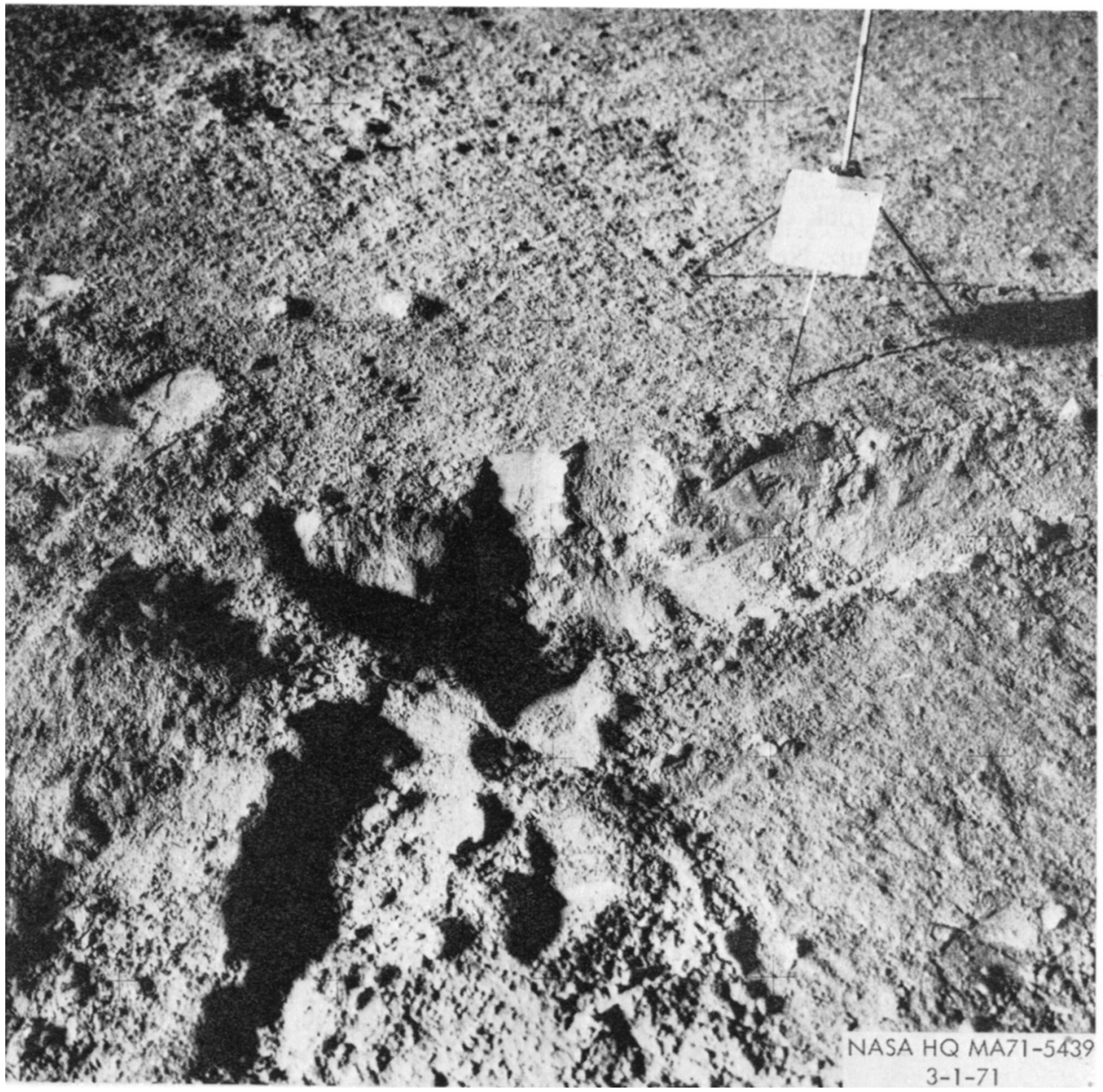

Fig. 9. Trench.

of the rocks were extensively documented by photography on the surface before being disturbed. Their highly diverse nature promises to hold an extremely interesting scientific story but one which will be difficult to unravel.

Figure 10 shows the location of the impacts of the spent stage of the launch vehicle and the Lunar Module when its primary job had been completed. These two impacts of known energy and location are important for attempting to understand the lunar interior from seismic signals.

While Captain Shepard and Commander Mitchell were on the surface, Major Roosa, in orbit, had a wide variety of tasks. Photography of potential future landing sites of the Descartes region was most important. In spite of the failure of a large camera, excellent photographs were taken with a back-up camera of the area of prime 
interest. Numerous other photographs of scientific interest were taken. Figure 11 shows some linear rilles in the Crater Fra Mauro. One can see a line of volcanic domes and apparently a flow of some type which may have obscured the rille in the vicinity. This area might possibly represent the source of seismic signals or gaseous emissions. It is far too early to consider this more than mere conjecture. Another interesting feature on an orbital photograph is shown in Figure 12. It is similar to a sinuous rille but is a positive feature with a peculiar cross section. A large number of additional scientific photographs were taken which will be the subject of further study.

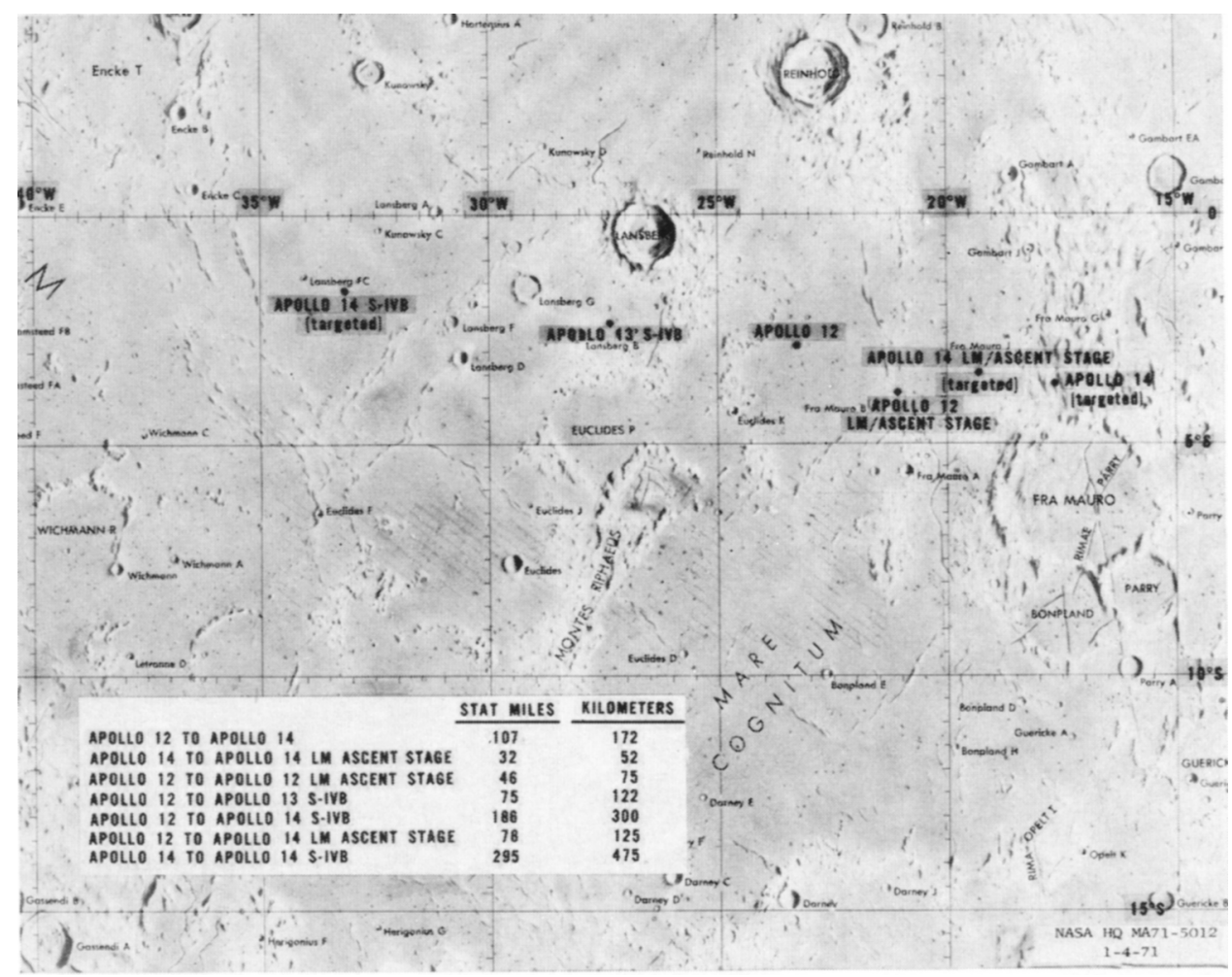

Fig. 10. Lunar impact locations.

On this mission, orbital tasks included low light level astronomical photography. The geometry of some of these tasks is indicated in Figures 13, 14, and 15. No results from this photography are as yet available. We know that photographs were made in the proper manner with the proper exposure but careful analysis with micro-densitometry equipment will be required to determine the results.

Another orbital experiment conducted was the recording of signals emanating from spacecraft and reflected from the lunar surface in the S-band and in the VHF fre- 
quencies. By comparing direct with reflected signals this bistatic radar data will give us information on the surface and near-surface roughness. With this experiment results are not as yet available but the data appear to be very good.

As a final point regarding the mission of Apollo 14, Figure 16 was constructed to show the progressive growth of scientific capabilities with each of the Apollo missions thus far. What is most significant is that these increases are not the result of major changes in hardware but rather results from increases in confidence and using what we have learned to improve procedures and techniques.

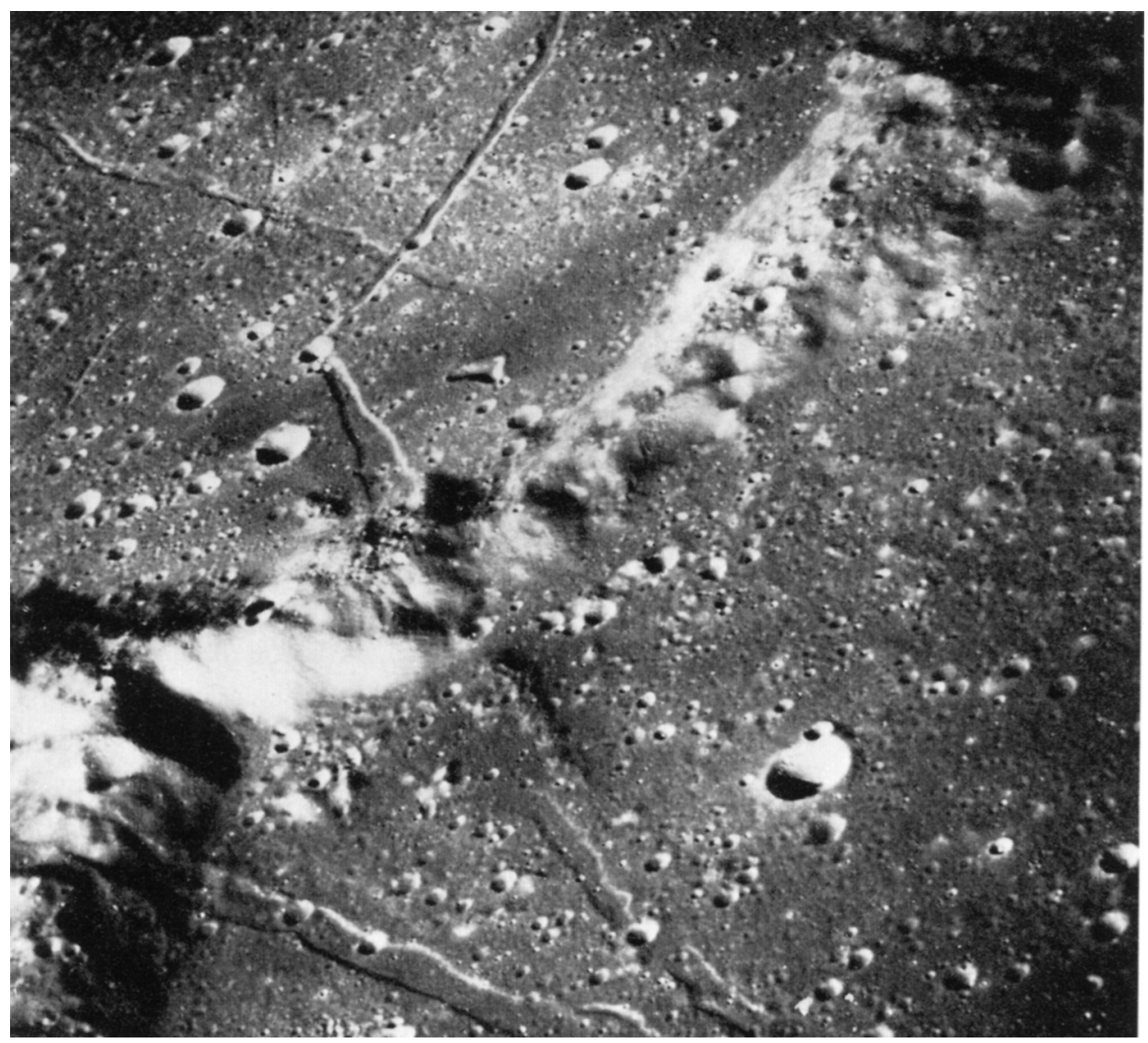

Fig. 11. Linear Rilles in crater Fra Mauro. 


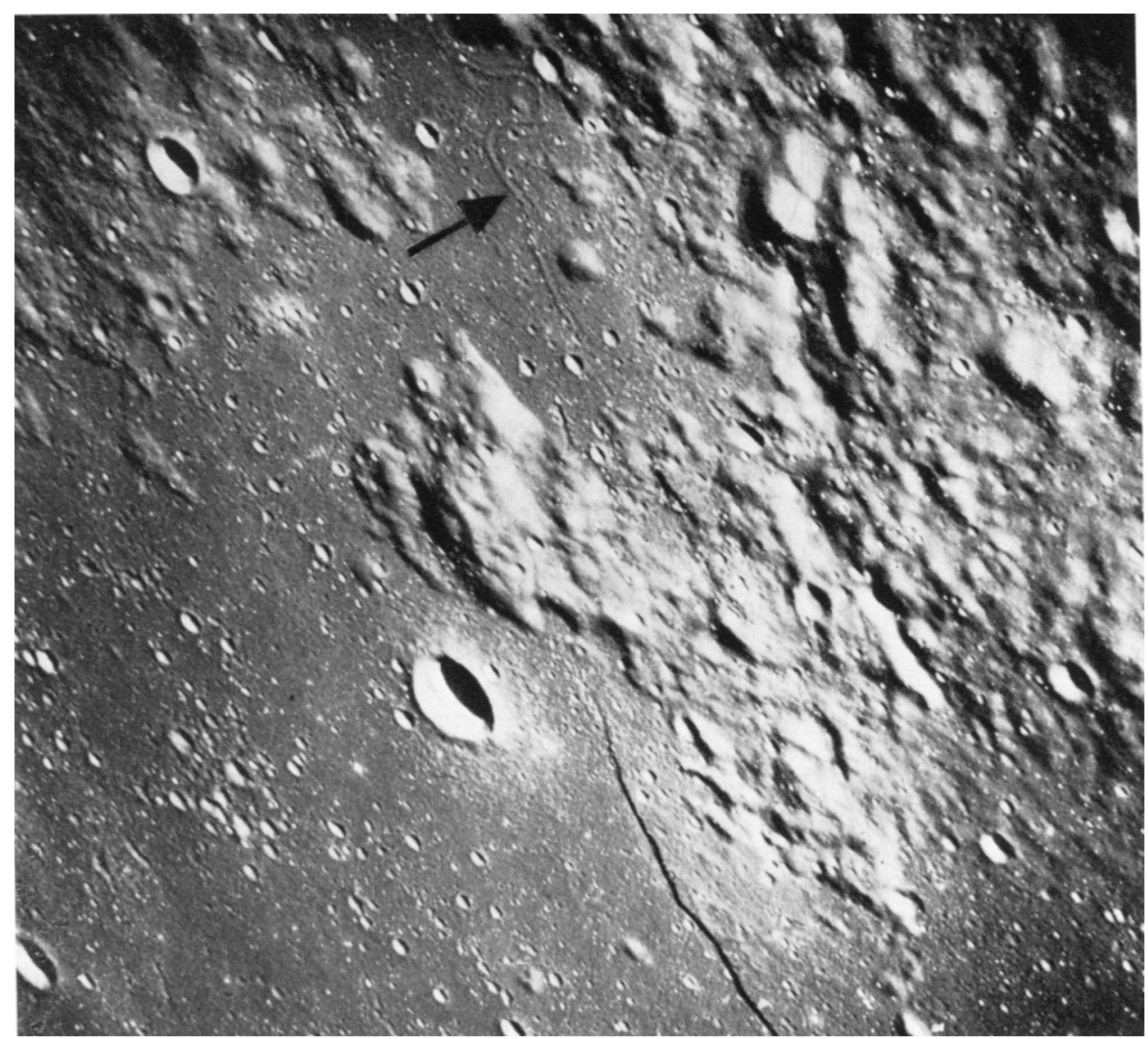

Fig. 12. Positive sinuous feature. 


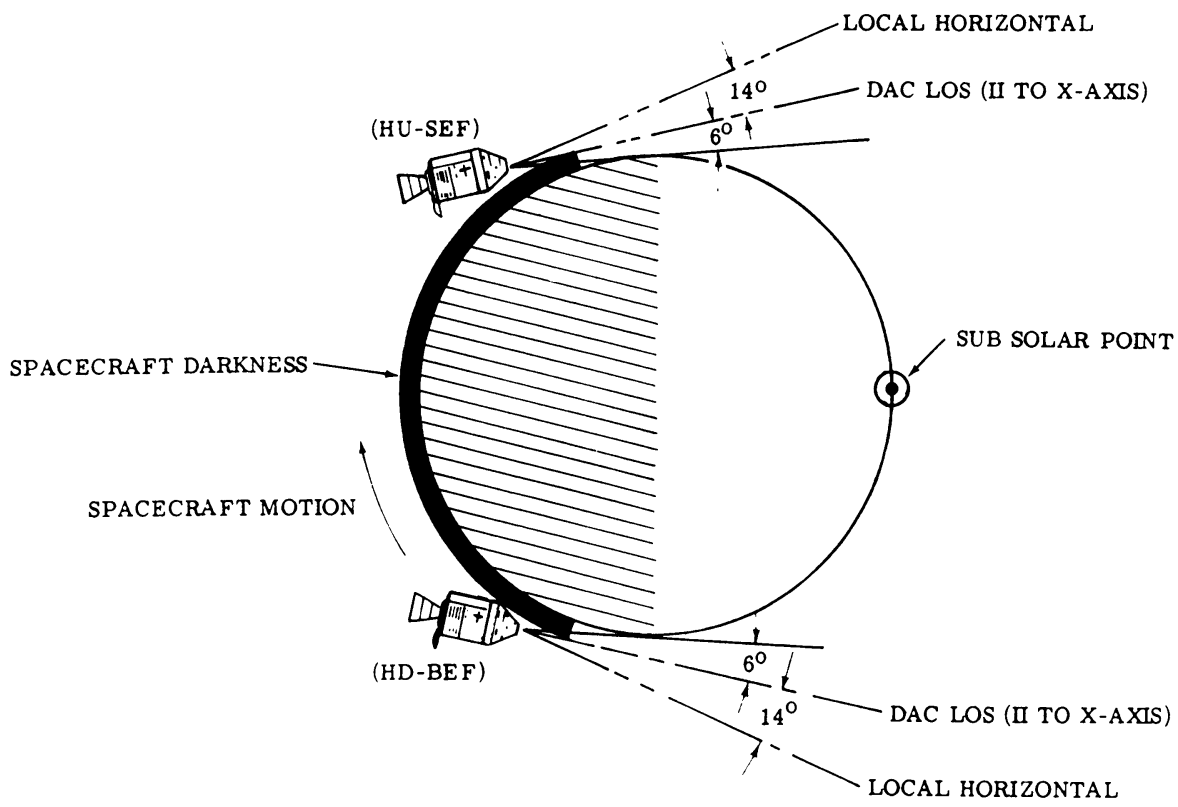

Fig. 13. Zodical light photography.

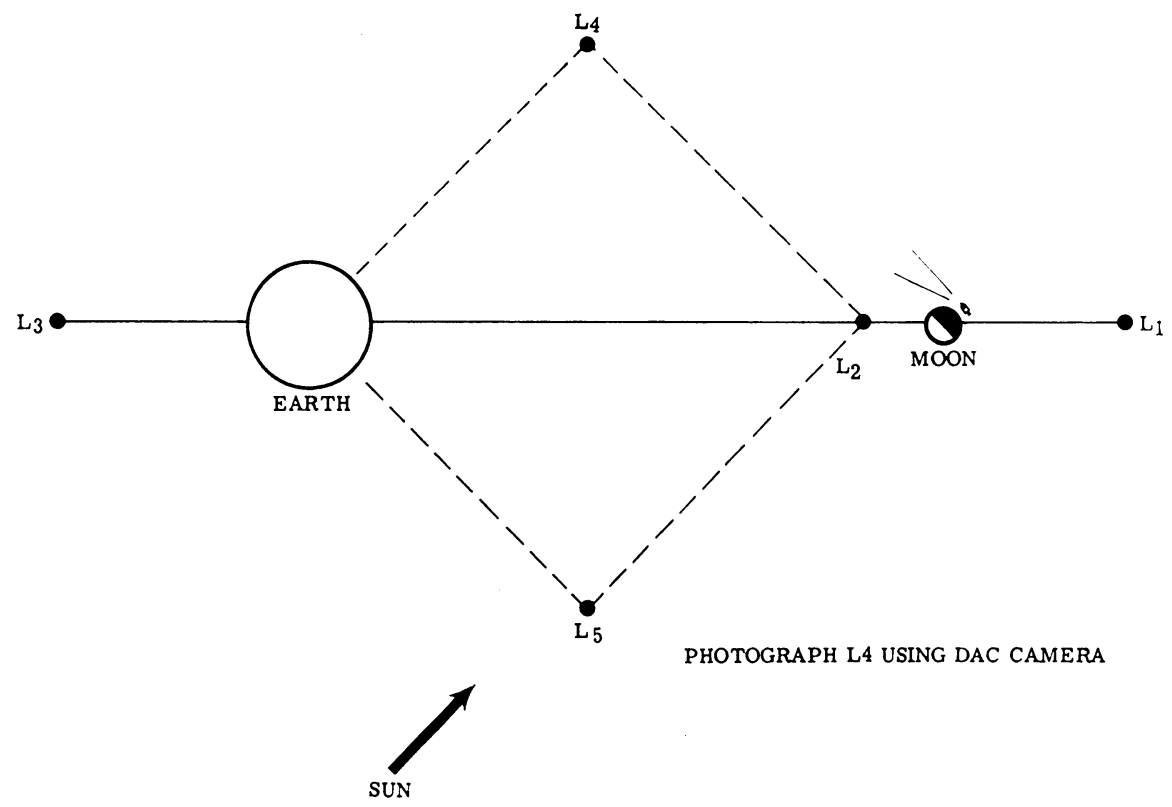

$A D O: 48745$

Fig. 14. Apollo 14 libration point photography. 


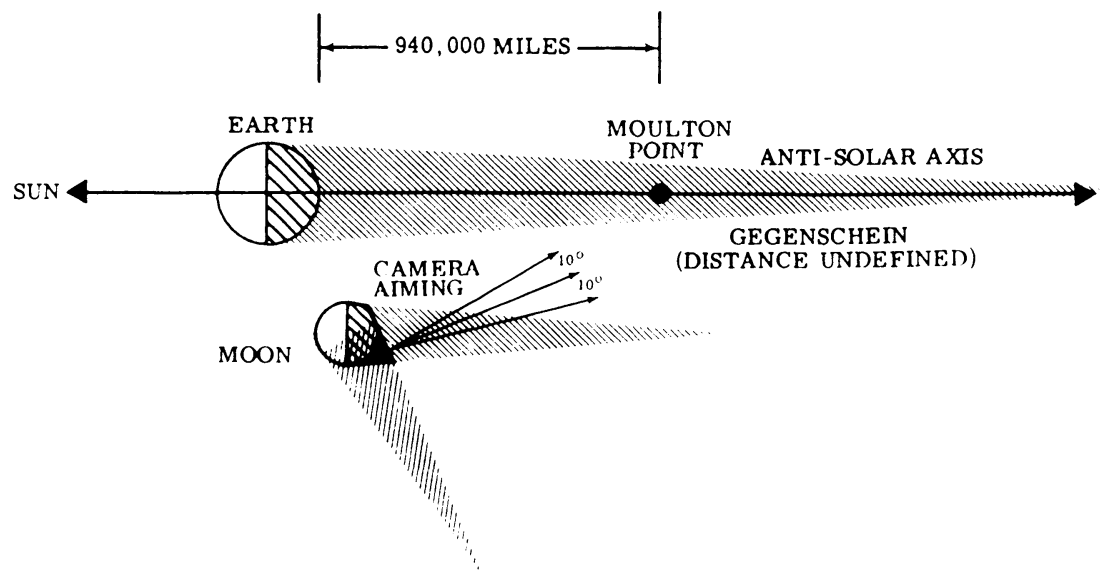

Fig. 15. Gegenschein experiment.

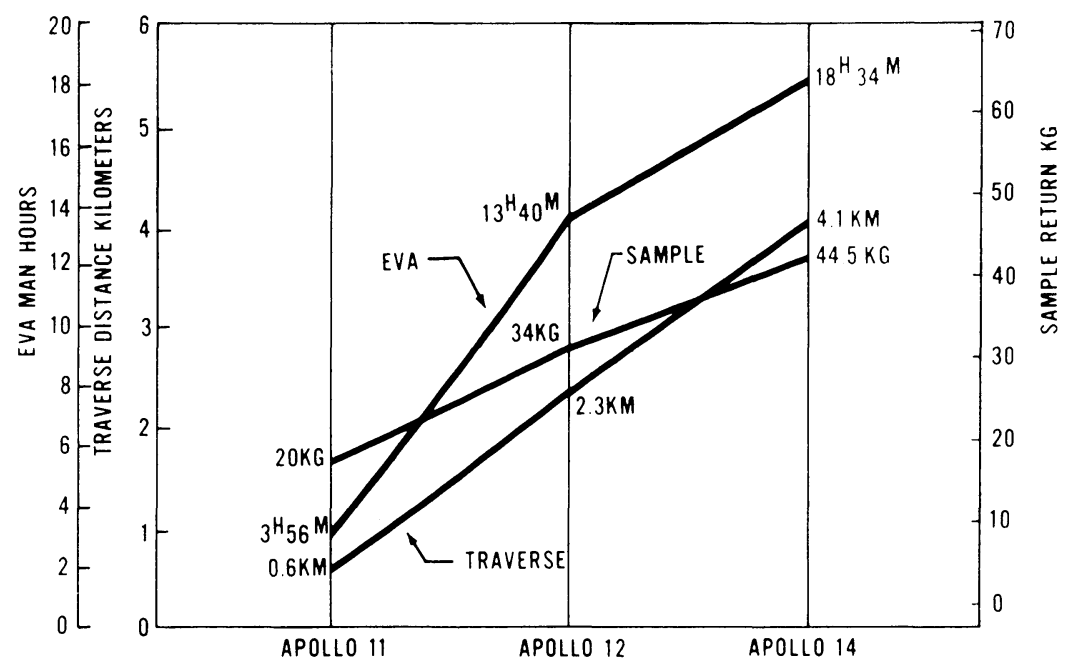

4086

Fig. 16. Mission science growth. 